\title{
INVESTIGACION
}

\section{Flavour changes due to effect of different packaging materials on storing of cottonseed oil, hydrogenated oil and margarine}

\author{
By Y. El-Shattory, M. A. Saadia and F. H. Said \\ Department of Fats and Oils, National Research Centre, Dokki, Cairo, Egypt
}

RESUMEN

Cambios en el flavor debidos al efecto de diferentes materiales de envases en almacenamiento de aceite de semilla de algodón, aceite hidrogenado y margarina.

Aceite de semilla de algodón decolorado, aceite de palma parcialmente hidrogenado y margarina fueron envasados en latas metálicas y botellas de plástico blancas y almacenados durante siete meses a temperatura ambiente en estantes. Se relacionó la evaluación de la estabilidad de los aceites con la deterioración del flavor. La deterioración del flavor producida en aceite de semilla de algodón decolorado, aceite de palma parcialmente hidrogenado y margarina fue observada debido al aumento en la cantidad de aldehídos y cetonas que juegan un importante papel en la deterioración de los aceites. A partir de los resultados obtenidos se encontró que las latas metálicas ofrecieron una adecuada y mejor protección frente a la deterioración que los envases de plásticos para aceite de semilla de algodón y aceite de palma parcialmente hidrogenado. Por otra parte, los recipientes de plástico fueron mejores para el almacenamiento de margarina y esto es debido a la presencia de agua y sales en ella en una proporción del $16 \%$ y $2 \%$ respectivamente.

PALABRAS-CLAVE: Aceite de semilla de algodón - Aceite hidrogenado - Almacenamiento - Envase - Flavor - Margarina.

\section{SUMMARY}

Flavour changes due to effect of different packaging materials on storing of cottonseed oil, hydrogenated oil and margarine.

Bleached cottonseed oil, partially hydrogenated palm oil and margarine were packed in metal cans and white plastic bottles and stored for seven months at room temperature on side bench. Assessment of the stability of the oils towards flavour deterioration was reported. The deterioration of flavour developed from bleached cottonseed oil, partially hydrogenated palm oil and margarine was observed due to increase amount of aldehydes and ketones which play an important role in deterioration of the oils. From the results we found metal can offered suitable and better protection against deterioration than plastic package for cottonseed oil, partially hydrogenated palm oil. While plastic container was better for storing margarine and this is due to the presence of water and salt in margarine where they compose about $16 \%$ and $2 \%$ respectively.

KEY-WORDS: Cottonseed oil - Flavour - Hydrogenated oil Margarine - Package - Storage.

\section{INTRODUCTION}

Exposure to diffuse daylight or artificial light is well known to cause a marked acceleration in the deterioration of unsaturated oil. This deterioration is probably sensitized by chromophoric impurities of oils, especially residual natural dyes and pigments, which absorbed strongly in visible or near ultraviolet light (1-3) and cause significant deterioration of oil quality in the presence of air (4-9).

Volatile oxidation products responsible for flavour alteration were studied by many authors (10-13) who found that fatty substances underwent autoxidation to produce small quantities of low molecular weight aldehydes, ketones and acids which have very objective odour and taste. This piece of work was done to study the effect of packing materials on storing of lipids.

\section{MATERIALS AND METHODS}

Bleached cottonseed oil, partially hydrogenated palm oil and margarine were stored in metal cans and white plastic bottles with air in the headspace and served at room temperature during the whole periode of investigation.

Suitable amount of aroma -concentration was prepared according to the method proposed by Sattar Co-workers (14).

The method of preparation of aroma concentrated was carried out by heating the samples on silicon oil 
bath at $190^{\circ} \mathrm{C}$ but for margarine at $135^{\circ} \mathrm{C}$ in $500 \mathrm{ml}$ round bottom flask under reduced pressure (about 30 $\mathrm{mm} \mathrm{Hg}$ ) for two hours. The aroma was collected in traps cooled to $-20^{\circ} \mathrm{C}$ using crushed ice-salt acetone mixture. The collected distillated was thrice extracted with peroxide-free ether/and the combined extracts were dried over anhydrous sodium sulphate. The extract was filtered and ether was then removed by distillation at $40^{\circ} \mathrm{C}$ to obtain the aroma concentrate.

Flavour of cottonseed oil, partially hydrogenated palm oil and margarine was detected at zero time (control), four and seven months storing by using gas liquid chromatography, the analysis was run using varian $\mathbf{3 7 0 0}$ gas chromatograph, equipped with flame ionization detector under the following conditions:

- Column package $10 \%$ diethylene glycol succinate (DEGS) on chromosorb W (80-100 mesh).
- Column length 6 feet with internal diameter $1 / 4$ inch.

- Column temperature: for flavour $70-190^{\circ} \mathrm{C}$ with programming rate $4^{\circ} \mathrm{C} / \mathrm{min}$.

- Injection temperature $220^{\circ} \mathrm{C}$.

- Detector temperature $300^{\circ} \mathrm{C}$.

- Carrier gas (He) flow rate $30 \mathrm{ml} / \mathrm{min}$.

- Hydrogen flow rate $30 \mathrm{ml} / \mathrm{min}$.

- Air flow rate $300 \mathrm{ml} / \mathrm{min}$. $(15,16)$

\section{RESULTS AND DISCUSSION}

Volatile components of control sample (zero time storing), were found to be composed of butanoic acid, 2-hexanol, methyl valerate, hexanoic acid, methyl hexanoate, heptanal and 3-ethyl hexanone (Table I).

Table I

\section{Percentage of Volatile Components of Cottonseed Oil}

\begin{tabular}{|c|c|c|c|c|c|c|c|}
\hline \multirow[t]{2}{*}{ Peak no. } & \multirow[t]{2}{*}{ tR } & \multirow[t]{2}{*}{ Control } & \multicolumn{2}{|c|}{ Metal can } & \multicolumn{2}{|c|}{ White plastic package } & \multirow[t]{2}{*}{ Component } \\
\hline & & & $4^{\text {th }}$ month & $7^{\text {th }}$ month & $4^{\text {th }}$ month & $7^{\text {th }}$ month & \\
\hline 1 & 0.5 & - & - & - & - & - & Ethanol \\
\hline 2 & 1.0 & - & - & - & - & - & Methyl ketone \\
\hline 3 & 1.5 & - & - & - & - & - & Ethyl ketone \\
\hline 4 & 2.0 & 0.99 & Trace & 20.23 & 13.21 & 13.13 & Propanal \\
\hline 5 & 2.5 & - & - & - & - & - & Butanal \\
\hline 6 & 2.75 & - & Trace & 3.52 & 14.10 & 7.41 & Pentanal \\
\hline 7 & 3.25 & 7.09 & - & 5.30 & - & 3.15 & Butanoic acid \\
\hline 8 & 3.75 & - & 3.1 & - & - & - & 1-Hexanol \\
\hline 9 & 4.5 & 12.41 & 34.1 & 39.22 & 5.53 & 18.20 & 2-Hexanol \\
\hline 10 & 5.5 & - & - & - & - & - & Unidentified \\
\hline 11 & 6.0 & - & - & - & - & - & Hexanal \\
\hline 12 & 6.5 & 2.13 & - & - & - & - & Unidentified \\
\hline 13 & 7.0 & 12.25 & - & 2.51 & - & 1.50 & Methyl valerate \\
\hline 14 & 7.5 & 17.14 & - & - & - & - & Hexanoic acid \\
\hline 15 & 8.0 & 17.42 & - & 8.00 & - & 4.21 & Methyl hexanoate \\
\hline 16 & 9.0 & 6.03 & - & 5.21 & - & 3.0 & Heptanal \\
\hline 17 & 9.5 & - & - & - & - & - & Heptanol \\
\hline 18 & 10.0 & - & - & - & - & - & Unidentified \\
\hline 19 & 10.5 & 3.25 & - & 1.50 & - & - & Octanal \\
\hline 20 & 11.25 & - & - & - & - & - & 1-Octanol \\
\hline 21 & 11.75 & - & - & - & - & - & Methyl heptanoate \\
\hline 22 & 12.5 & - & - & - & - & - & Methyl octanoate \\
\hline 23 & 13.0 & - & - & - & - & - & Unidentified \\
\hline 24 & 13.75 & - & - & - & - & - & Unidentified \\
\hline 25 & 14.5 & 0.75 & 3.4 & 2.61 & 2.32 & 1.4 & Nonanal \\
\hline 26 & 15.5 & - & - & - & - & - & - Unidentified \\
\hline 27 & 16.0 & - & - & 6.6 & - & 3.33 & Decanal \\
\hline 28 & 18.0 & 1.38 & - & 0.9 & - & - & 1-Octene-3-ol \\
\hline 29 & 18.5 & 0.35 & 7.2 & 0.3 & - & - & Hendecanal \\
\hline 30 & 19.0 & - & - & - & - & - & Unidentified \\
\hline 31 & 19.5 & 0.53 & - & - & 8.1 & 3.10 & Octanol \\
\hline 32 & 20.0 & - & - & - & - & - & Unidentified \\
\hline 33 & 20.5 & 3.33 & - & 0.9 & - & - & Dodecanal \\
\hline
\end{tabular}


Table I Continue

Percentage of Volatile Components of Cottonseed Oil

\begin{tabular}{cccccccc}
\hline Peak no. & tR & Control & \multicolumn{2}{c}{ Metal can } & \multicolumn{2}{c}{ White plastic package } & Component \\
& & & 4th $^{\text {th }}$ month & 7th $^{\text {th }}$ month & 4h $^{\text {th }}$ month & 7th $^{\text {month }}$ & \\
\hline 34 & 22.0 & 0.28 & - & - & - & - & Decanoic acid \\
35 & 23.0 & 0.89 & 4.1 & 0.8 & 5.2 & 5.27 & Nonenal \\
36 & 24.5 & 0.71 & - & - & 1.3 & - & 2,5-Hexadione \\
37 & 25.1 & 0.39 & 4.6 & 2.4 & 10.6 & 7.30 & 2,4-Nonadienal \\
38 & 25.5 & 7.37 & 3.1 & - & - & - & 3-Ethyl hexanone \\
39 & 29.0 & 2.24 & 3.9 & - & 10.2 & 7.5 & 3-Ethyl heptanone \\
40 & 30.5 & 3.07 & - & - & 1.5 & - & 3-Ethyl octanone \\
41 & 31.5 & - & - & - & 5.12 & 6.2 & 3-Ethyl nonanone \\
42 & 33.0 & - & 9.20 & - & 4.31 & 2.30 & 2,4-Decadienal \\
43 & 35.5 & - & 27.30 & - & 15.20 & 9.00 & Undecanol \\
44 & 37.5 & - & - & - & 3.31 & 4.00 & Unidentified \\
\hline
\end{tabular}

These volatile components constituted more than $79 \%$ of the total volatile components of bleached cottonseed oil at zero time storing.

In metal can backage 2-hexanol increased after four and seven months, on the other side butanoic acid, methyl valerate, hexanoic acid, methyl hexanoate and heptanal completely disappeared after storing for four months for metal can and plastic containers propanal, pentanal, 2-hexanol, 2-4, nonadienal and undecanol formed about $66.0 \%$ and $58.0 \%$ after storage for four months in metal can and plastic container respectively, while they composed $65.6 \%$ and $55.0 \%$ after storage for seven months in both packages, respectively.

The deterioration of flavour developed from hydrogenated oil is reported in (Table II). A remarkable oxidation was observed due to increase amount of hexanal. Ketones played an important role in the flavour of partially hydrogenated palm oil. Alcohols and ester were also identified in the aroma of partially hydrogenated palm oil.

The changes in the flavour developed from margarine stored in metal can and white plastic package for four and seven months compared with control sample were reported in (Table III).

A remarkable oxidation was observed due to increase of aldehydes and some ketones. Short chain fatty acids were probably produced by chain breaking through autoxidation and they play a major role in the odour of margarine. Alcohols generally played a minor role in the aroma of margarine.

Table II

Percentage of Volatile Components of Partially Hydrogenated Palm Oil

\begin{tabular}{|c|c|c|c|c|c|c|c|}
\hline \multirow[t]{2}{*}{ Peak no. } & \multirow[t]{2}{*}{ tR } & \multirow[t]{2}{*}{ Control } & \multicolumn{2}{|c|}{ Metal can } & \multicolumn{2}{|c|}{ White plastic package } & \multirow[t]{2}{*}{ Component } \\
\hline & & & $4^{\text {th }}$ month & $7^{\text {th }}$ month & $4^{\text {th }}$ month & $7^{\text {th }}$ month & \\
\hline 1 & 0.5 & - & - & - & - & - & Ethanol \\
\hline 2 & 1.0 & - & - & - & - & - & Methyl ketone \\
\hline 3 & 1.5 & - & - & - & - & - & Ethyl ketone \\
\hline 4 & 2.0 & - & - & - & - & - & Propanal \\
\hline 5 & 2.5 & - & - & - & - & - & Butanal \\
\hline 6 & 2.75 & - & - & - & - & - & Pentanal \\
\hline 7 & 3.25 & - & - & - & - & - & Butanoic acid \\
\hline 8 & 3.75 & - & - & - & - & - & 1-Hexanol \\
\hline 9 & 4.50 & 20.59 & 28.32 & 30.13 & 16.41 & 17.61 & 2-Hexanol \\
\hline 10 & 5.5 & - & - & 7.5 & - & - & Unidentified \\
\hline 11 & 6.0 & 3.59 & - & - & - & 4.30 & Hexanal \\
\hline 12 & 6.5 & - & - & - & - & - & Unidentified \\
\hline 13 & 7.0 & - & - & - & - & - & Methyl valerate \\
\hline 14 & 7.5 & - & - & - & - & - & Hexanoic acid \\
\hline
\end{tabular}


Table II (Continue)

Percentage of Volatile Components of Partially Hydrogenated Palm Oil

\begin{tabular}{|c|c|c|c|c|c|c|c|}
\hline \multirow[t]{2}{*}{ Peak no. } & \multirow[t]{2}{*}{ tR } & \multirow[t]{2}{*}{ Control } & \multicolumn{2}{|c|}{ Metal can } & \multicolumn{2}{|c|}{ White plastic package } & \multirow[t]{2}{*}{ Component } \\
\hline & & & $4^{\text {th }}$ month & $7^{\text {th }}$ month & $4^{\text {th }}$ month & $7^{\text {th }}$ month & \\
\hline 15 & 8.0 & - & - & - & - & - & Methyl hexanoate \\
\hline 16 & 9.0 & - & - & - & - & - & Heptanal \\
\hline 17 & 9.5 & - & - & - & - & - & Heptanol \\
\hline 18 & 10.0 & - & - & - & - & - & Unidentified \\
\hline 19 & 10.5 & - & - & - & - & - & Octanal \\
\hline 20 & 11.25 & - & - & - & - & - & 1-Octanol \\
\hline 21 & 11.75 & - & - & - & - & - & Methyl heptanoate \\
\hline 22 & 12.5 & 17.10 & 9.24 & 4.7 & 19.00 & 10.52 & Methyl octanoate \\
\hline 23 & 13.0 & - & - & - & - & - & Unidentified \\
\hline 24 & 13.75 & 2.82 & - & 1.90 & 3.52 & 4.00 & Unidentified \\
\hline 25 & 14.5 & - & - & - & - & - & Nonanal \\
\hline 26 & 15.5 & 2.25 & Trace & 1.34 & 2.97 & 3.21 & Unidentified \\
\hline 27 & 16.0 & - & - & - & - & - & Decanal \\
\hline 28 & 18.0 & - & - & - & - & - & 1-Octene-3-ol \\
\hline 29 & 18.5 & - & - & - & - & - & Hendecanal \\
\hline 30 & 19.0 & - & - & - & - & - & Unidentified \\
\hline 31 & 19.5 & - & - & - & - & - & Octanol \\
\hline 32 & 20.0 & - & - & - & - & - & Unidentified \\
\hline 33 & 20.5 & - & - & - & - & - & Dodecanal \\
\hline 34 & 22.0 & 1.56 & Trace & 1.5 & 2.0 & 2.2 & Decanoic acid \\
\hline 35 & 23.0 & 15.58 & 20.2 & 19.53 & 17.9 & 18.1 & Nonenal \\
\hline 36 & 24.5 & 5.10 & 5.1 & 5.1 & 7.3 & 8.0 & 2,5-Hexadione \\
\hline 37 & 25.1 & 25.45 & 21.2 & 21.3 & 21.4 & 21.21 & 2,4-Nonadienal \\
\hline 38 & 25.5 & 3.89 & 5.31 & 4.66 & 5.36 & 6.12 & 3-Ethyl hexanoate \\
\hline 39 & 29.0 & 2.07 & 3.20 & 2.30 & 4.14 & 4.73 & 3-Ethyl heptanone \\
\hline 40 & 30.5 & - & - & - & - & - & 3-Ethyl octanone \\
\hline 41 & 31.5 & - & - & - & - & - & 3-Ethyl nonanone \\
\hline 42 & 33.0 & - & - & - & - & - & 2,4-dodecadienal \\
\hline 43 & 35.5 & - & - & - & - & - & Undecanol \\
\hline 44 & 37.5 & - & - & - & - & - & Unidentified \\
\hline
\end{tabular}

Table III

Percentage of Volatile of Margarine

\begin{tabular}{|c|c|c|c|c|c|c|c|}
\hline \multirow[t]{2}{*}{ Peak no. } & \multirow[t]{2}{*}{ tR } & \multirow[t]{2}{*}{ Control } & \multicolumn{2}{|c|}{ Metal can } & \multicolumn{2}{|c|}{ White plastic package } & \multirow[t]{2}{*}{ Component } \\
\hline & & & $4^{\text {th }}$ month & $7^{\text {th }}$ month & $4^{\text {th }}$ month & $7^{\text {th }}$ month & \\
\hline 1 & 0.5 & - & - & - & - & - & Ethanol \\
\hline 2 & 1.0 & 0.11 & 2.2 & 3.2 & 2.1 & 3.3 & Methyl ketone \\
\hline 3 & 1.5 & - & 0.9 & 1.2 & Trace & 0.1 & Ethyl ketone \\
\hline 4 & 2.0 & 0.13 & 2.9 & 3.0 & 1.5 & 2.3 & Propanal \\
\hline 5 & 2.5 & - & - & - & - & - & Butanal \\
\hline 6 & 2.75 & - & - & - & - & - & Pentanal \\
\hline 7 & 3.25 & 3.21 & 6.9 & 6.1 & 4.6 & 5.2 & Butanoic acid \\
\hline 8 & 3.75 & - & - & - & - & - & 1-Hexanol \\
\hline 9 & 4.5 & 16.0 & 13.5 & 15.6 & 17.2 & 16.4 & 2-Hexanol \\
\hline 10 & 5.5 & - & - & - & - & - & Unidentified \\
\hline 11 & 6.0 & 1.21 & 2.1 & 2.2 & 1.5 & 2.3 & Hexanal \\
\hline
\end{tabular}


Table III (Continue)

Percentage of Volatile of Margarine

\begin{tabular}{|c|c|c|c|c|c|c|c|}
\hline \multirow[t]{2}{*}{ Peak no. } & \multirow[t]{2}{*}{ tR } & \multirow[t]{2}{*}{ Control } & \multicolumn{2}{|c|}{ Metal can } & \multicolumn{2}{|c|}{ White plastic package } & \multirow[t]{2}{*}{ Component } \\
\hline & & & $4^{\text {th }}$ month & $7^{\text {th }}$ month & $4^{\text {th }}$ month & $7^{\text {th }}$ month & \\
\hline 12 & 6.5 & 0.99 & 1.00 & 1.2 & Trace & Trace & Unidentified \\
\hline 13 & 7.0 & 5.20 & 5.5 & 3.2 & 4.9 & 4.3 & Methyl valerate \\
\hline 14 & 7.5 & 6.10 & 5.5 & 3.7 & 4.9 & 3.97 & Hexanoic acid \\
\hline 15 & 8.0 & 6.32 & 6.1 & 4.2 & 5.12 & 4.86 & Methyl hexanoate \\
\hline 16 & 9.0 & 2.03 & 3.2 & 3.1 & 3.0 & 3.40 & Heptanal \\
\hline 17 & 9.5 & - & 1.8 & 2.2 & Trace & 2.0 & Heptanol \\
\hline 18 & 10.0 & - & - & - & - & - & Unidentified \\
\hline 19 & 10.5 & 1.01 & 2.0 & 2.3 & 1.04 & 1.9 & Octanal \\
\hline 20 & 11.25 & - & - & - & - & - & 1-Octanol \\
\hline 21 & 11.75 & - & - & - & - & - & Methyl heptanoate \\
\hline 22 & 12.5 & 5.82 & 5.8 & 4.2 & 5.1 & 4.3 & Methyl octanoate \\
\hline 23 & 13.0 & - & - & - & - & - & Unidentified \\
\hline 24 & 13.75 & 0.85 & 1.0 & - & Trace & 0.5 & Unidentified \\
\hline 25 & 14.5 & 0.12 & Trace & - & - & Trace & Nonanal \\
\hline 26 & 15.5 & 0.74 & 1.4 & 1.6 & 1.4 & 1.7 & Unidentified \\
\hline 27 & 16.0 & - & - & - & - & - & Decanal \\
\hline 28 & 18.0 & 0.62 & 1.1 & 2.2 & 1.1 & 1.2 & 1-Octen-3-ol \\
\hline 29 & 18.5 & 0.10 & 1.0 & 1.1 & Trace & 1.6 & Hendecanal \\
\hline 30 & 19.0 & - & - & - & - & - & Unidentified \\
\hline 31 & 19.5 & 0.16 & 1.2 & 1.5 & 0.99 & 1.5 & Octanol \\
\hline 32 & 20.0 & - & - & - & - & - & Unidentified \\
\hline 33 & 20.5 & 1.71 & Trace & 2.1 & 1.2 & 1.9 & Dodecanal \\
\hline 34 & 22.0 & 20.24 & 16.1 & 18.4 & 17.9 & 16.5 & Decanoic acid \\
\hline 35 & 23.0 & 7.66 & 3.5 & 4.2 & 7.4 & 4.3 & Nonenal \\
\hline 36 & 24.5 & 3.20 & 2.2 & 2.6 & 2.4 & 2.1 & 2,5-Hexadione \\
\hline 37 & 25.1 & 8.30 & 6.1 & 5.2 & 6.9 & 5.2 & 2,4-Nonadienal \\
\hline 38 & 25.5 & 5.07 & 3.0 & 3.0 & 4.1 & 3.3 & 3-Ethyl hexanone \\
\hline 39 & 29.0 & 1.80 & 2.4 & 1.2 & 2.9 & 2.8 & 3-Ethyl heptanone \\
\hline 40 & 30.5 & 1.30 & 1.6 & 1.5 & 2.7 & 3.0 & 3-Ethyl octanone \\
\hline 41 & 31.5 & - & - & - & - & - & 3-Ethyl nonanone \\
\hline 42 & 33.0 & - & - & - & - & - & 2,4-dodecadienal \\
\hline 43 & 35.5 & - & - & - & - & - & Undecanol \\
\hline 44 & 37.5 & - & - & - & - & - & Unidentified \\
\hline
\end{tabular}

Figure 1-4 show the characteristic volatile components of cottonseed oil, partially hydrogenated palm oil and margarine during storing period. From these figures we can trace:

- Propanal increased in both metal cans and white plastic package after $7^{\text {th }}$ month storage, while hexanal increase in both $4^{\text {th }}$ and $7^{\text {th }}$ months storage in two kinds of package in cottonseed oil.

- Decanoic acid was highly increased in margarine in both $4^{\text {th }}$ and $7^{\text {th }}$ months storage and in the two kinds of packaging materials.

- Nonenal and 2,4-nonadienal were highly increased in partially hydrogenated palm oil in both kings of package.
Generally from all these results it could be traced that propanal might arise from 1-decomposition of the hydroperoxide of linolenate, 2-stepwise oxidation of nnonanal through the $\mathrm{C}_{2-4}$ alkanals to propanal (10) and 3-oxidation of n-propanol with alcohol dehydrogenase.

Alcohols may be derived from unsaturated acids by the formation and reduction of the corresponding aldehydes (17). 2,4-nonadienal could be postulated as being formed from linoleic acid as proposed by Hoffmann (18). These results are in agreement with the peroxide value of bleaching cottonseed oil, partially hydrogenated palm pol and margarine (Table IV). 
Table IV

Peroxide values of different samples

\begin{tabular}{lccccc}
\hline \multicolumn{1}{c}{ Sample } & Control & \multicolumn{2}{c}{ Metan can } & \multicolumn{2}{c}{ White plastic } \\
& & $\mathbf{4}^{\text {th }}$ & 7 $^{\text {th }}$ & $\mathbf{4}^{\text {th }}$ & $\mathbf{7}^{\text {th }}$ \\
\hline Bleaching cottonseed oil & 0.4 & 16.3 & 35.1 & 19.0 & 24.0 \\
Partially hydrogenated palm oil & 4.1 & 20.3 & 45.5 & 24.2 & 35.0 \\
Margarine & 2.0 & 38.3 & 84.6 & 33.9 & 75.4 \\
\hline
\end{tabular}

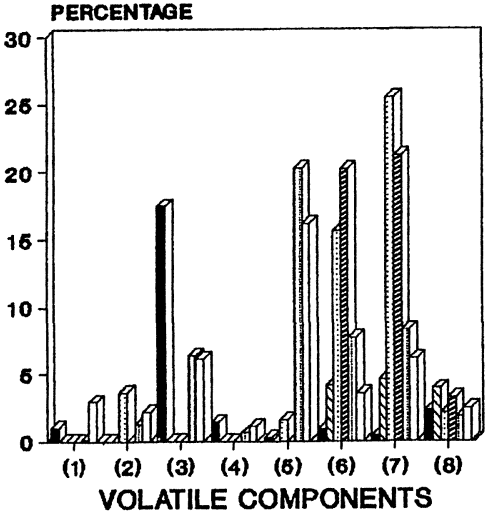

(1) Propanal

(4) 1-Octen-3-ol

(7) 2,4-Nonadienal

(2) Hexanal

(5) Decanoic acid

(8) Ethyl heptanone

(3) Methyl hexanoate

(6) Nonenal

Figure 1

Volatile components of cottonseed oil, partially hydrogenated palm oil and margarine stored in metal cans for four months

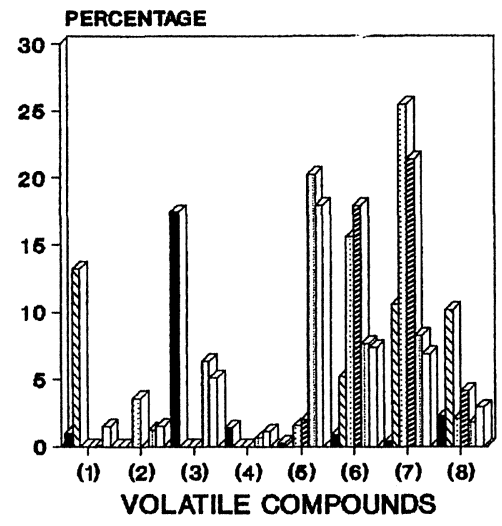
(1) Propanal
(4) 1-Octen-3-ol
(2) Hexanal
(7) 2,4-Nonadienal
(5) Decanoic acid
(8) Ethyl heptanone

(3) Methyl hexanoate (6) Nonenal

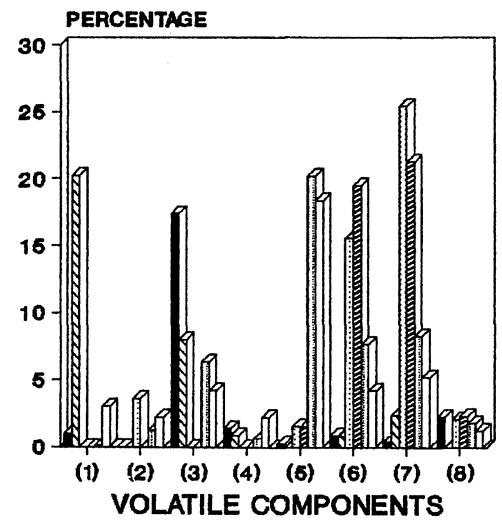

(1) Propanal
(4) 1-Octen-3-ol
(7) 2,4-Nonadienal

(2) Hexanal

(5) Decanoic acid

(8) Ethyl heptanoate
CONTROL,CSO

Ith month,cso

CONTROL,PHPO

7th month,PHPO

[iitili CONTROL,MARQARINE

$\square 7$ th month,MARGARINE

Figure 2

Volatile components of cottonseed oil, partially hydrogenated palm oil and margarine stored in metal cans for seven months

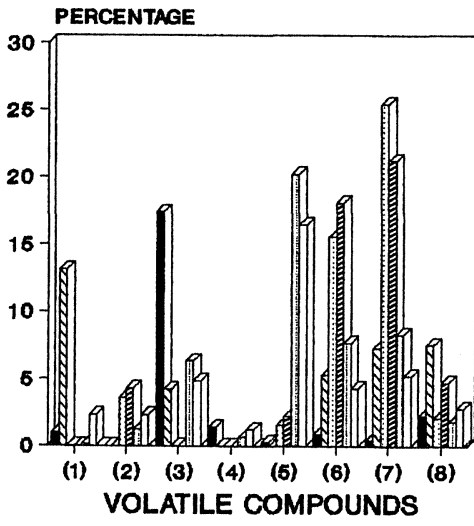

CONTROL,CSO

7th month,cso

CONTROL,PHPO

7th month,PHPO

iiㄹ CONTROL, MARGARINE

$\square$ 7th mOnth,MARGARINE
(2) Hexanal

(5) Decanoic acid

(8) Ethyl heptanone

Figure 3

Volatile components of cottonseed oil, partially hydrogenated palm oil and margarine stored in white plastic bottles for four months

Figure 4

Volatile components of cottonseed oil, partially hydrogenated palm oil and margarine stored in white plastic bottles for seven months. 


\section{REFERENCES}

1. Rawls, H. R., and Van Santen, P. J. (1970). - «Possible role for singlet oxygen in the initiation of fatty acid autoxidation".- J. Amer. Oil Chem. Soc. 47 (4), 121-125.

2. Chahine, M. H. and De Man, J. M. (1971). - «Autoxidation of corn oil under the influence of fluorescent light". - Can. Inst. Food Technol., J. 4 (1), 2428.

3. Clements, A. H., Vanden Engh, R. H., Frost, D. J. Hoogenhout, K., Nooi, J. R. (1973). -«Participation of singlet oxygen in photosensitized oxidation of 1,4-dienoic systems and photooxidation of soybean oil». -J. Amer. Oil Chem. Soc. 50 (8), 325-330.

4. Evans, C.D., List, G. R., Moser, H. A., and Cowan, J. C. (1973). - «Long term storage of soybean and cottonseed salad oils».- J. Amer. Oil Chem. Soc. 50 (6), 218-222.

5. Coleman. W. W., Watrous, G. H. and Dimick, P. S. (1976). - J. Milk Food Technol. 39, 55.

6. De Man, John M. and Sattar, Abdus (1975) -«Photooxidation of milk and milk products».- Review Food Science Nutr. 7 (1), 13-37.

7. Hansen, A. P., Turner, L. G. and Burand, L. W. (1975). -J. Milk Food Technol. 38, 388.

8. Moser, H. A., Evans, C. D. Cowan, J. C. and Kwolek, W. F. (1965). - «A light test to measure stability of edible oils".- J. Amer. Oil Chem. Soc. 42 (1), 30-33.

9. Sattar, A., De Man, J. M., Alexander, J. C. (1976). -Can. Inst. Food Sci. Technol. J. 9, 149.

10. Loury, M., Lerchartier, G., and Forney, M. (1965). - «Identification of volatile products of the autoxidation of oleic acid, by paper and thin-layer chromatography s-. Rev. Franc. Corps Gras 12 (4), 253-262.

11. Hill, F. D. and Hammond, E. G. (1965). -«Studies on the flavour of autoxidize soybean oil»-. J. Amer. Oil. Chem. Soc., 42, 1148-1150.

12. Sattar, A., De Man J. M. and Alexander, J. C. (1976). - «Stability of edible oils and fats to fluorescent light irradiation»-. J. Amer. Oil Chem. Soc. 53 (7), 473-477.

13. Sattar, A., De Man J. M., and Alexander, J. C. (1976). - Effect of wavelength on light induced quality deterioration of edible oils and fats»-. Inst. Food Sci., Technol. J. 9 (3), 108-113.

14. Sattar, A., De Man J. M. and Alexander, J. C. (1976). - «Light induced oxidation of edible oils and fats»-. Lebensm, Wiss U-Technol. J. 9 (3), 149-152.

15. Ghan, H. W. S., Levett, G. and Griffiths, N. M. (1977). -J. Sci. Food Agric. 28, 339.

16. O'Brien, J. S., Fillerup, D. L. and Mead, J. F. (1964). - QQuantification and fatty acid and fatty aldehyde composition of ethanolamine, choline and serine glycerophosphatides in human cerebral gray and white matter»-. J. Lipid Res., 5 (3), 329-338.

17. Guymon, J. F. (1964). - «Studies of higher alcohol formation by yeasts through gas chromatography"-. Qualitas Plant. Mater. Vegetabiles 11, 194-201.

18. Hoffmann, G. (1962). -J. Amer. Oil Chem. Soc., 39, 439. 